\title{
Phase structure of an Abelian two-Higgs model and high temperature superconductors
}

\author{
M. N. Chernodub, ${ }^{1,2}$ E.-M. Ilgenfritz, ${ }^{3}$ and A. Schiller ${ }^{4}$ \\ 1 ITEP, B.Cheremushkinskaya 25, RU-117259 Moscow, Russia \\ ${ }^{2}$ Department of Theoretical Physics, Uppsala University, P.O. Box 803, S-75108, Uppsala, Sweden \\ ${ }^{3}$ Institut für Physik, Humboldt-Universität zu Berlin, Newtonstr. 15, D-12489 Berlin, Germany \\ ${ }^{4}$ Institut für Theoretische Physik, Universität Leipzig, D-04109 Leipzig, Germany
}

\begin{abstract}
We study the phase structure of a three dimensional Abelian Higgs model with singly- and doublycharged scalar fields coupled to a compact Abelian gauge field. The model is pretending to describe systems of strongly correlated electrons such as high- $T_{c}$ superconductivity in overdoped regime and exotic phases supporting excitations with fractionalized quantum numbers. We identify the Fermi liquid, the spin gap, the superconductor and the strange metallic phases in which densities and properties of holon and spinon vortices and monopoles are explored. The phase diagram in the $3 D$ coupling space is predicted. We show that at sufficiently strong gauge coupling the spinon-pair and holon condensation transitions merge together and become, unexpectedly, first order.
\end{abstract}

PACS numbers: 74.10.+v,71.10.Hf,11.15.Ha,74.90.+n

The physics of high- $T_{c}$ superconductivity [1, 2] is not yet completely understood. However, certain outstanding features are generally recognized. At normal temperatures, all known high- $T_{c}$ superconductors are ceramic materials characterized by a poor conductivity. At low temperatures the clean material is a Mott insulator. Doping it with impurities, the insulator may become a superconductor at low enough temperatures. The physics of high- $T_{c}$ superconductivity is essentially a $2 D$ phenomenon since charge carriers - which are electrons or holes provided by the dopants - are confined to the $\mathrm{CuO}_{2}$ planes typical for all high- $T_{c}$ superconductors.

A popular approach to high- $T_{c}$ superconductivity is provided by the $t-J$ Hamiltonian [3] describing hopping holes and localized spins in a plane:

$$
H_{t J}=-t \sum_{<i j>, \sigma} c_{i \sigma}^{\dagger} P_{i j,-\sigma} c_{j \sigma}+J \sum_{<i j>}\left(\vec{S}_{i} \vec{S}_{j}-\frac{1}{4} n_{i} n_{j}\right)
$$

The first term describes electrons moving without changing spin $\sigma$. Double occupancy is explicitly forbidden by the presence of the projectors $P_{i j, \sigma}=\left(1-n_{i, \sigma}\right)\left(1-n_{j, \sigma}\right)$. The second term specifies the anti-ferromagnetic Heisenberg coupling between spins located at the copper sites. Here $\vec{S}_{i}=(1 / 2) \sum_{\sigma \sigma^{\prime}} c_{i \sigma}^{\dagger} \vec{\tau}_{\sigma \sigma^{\prime}} c_{i \sigma^{\prime}}$ is the spin operator, $c_{i \sigma}^{\dagger}$, $c_{i \sigma}$ are the hole (or electron) creation and annihilation operators, $n_{i, \sigma}=c_{i \sigma}^{\dagger} c_{i \sigma}$ denotes the occupation number, and $n_{i}=n_{i, \uparrow}+n_{i, \downarrow}$.

The $t$ - $J$ model (11) is often treated in the slave-boson technique [4], which splits the spin and charge degrees of freedom of the electrons. The electron creation operators are decomposed as [4, 5] $c_{i \sigma}^{\dagger}=f_{i \sigma}^{\dagger} b_{i}$, where $f_{i \sigma}$ is a spin-particle ("spinon") operator and $b_{i}$ is a charge-particle ("holon") operator. In order to forbid double occupancy of sites one imposes the constraint $f_{i \uparrow}^{\dagger} f_{i \uparrow}+f_{i \downarrow}^{\dagger} f_{i \downarrow}+b_{i}^{\dagger} b_{i}=1$ on the physical states of the system. The constraint drastically simplifies [5] the treatment of the $t-J$ Hamiltonian (1).
In addition to the ordinary electromagnetic (external) gauge symmetry, the spin-charge separation naturally introduces an (internal) compact $U(1)$ gauge freedom,

$$
U(1)_{\mathrm{int}}: \quad c_{i \sigma} \rightarrow c_{i \sigma}, \quad f_{i \sigma} \rightarrow e^{i \alpha_{i}} f_{i \sigma}, \quad b_{i} \rightarrow e^{i \alpha_{i}} b_{i \sigma}
$$

which plays an essential role [6] in understanding the physics of strongly correlated electrons. Besides condensed matter physics, the spin-charge separation idea is also applied to strongly interacting gluons in QCD [7].

The emerging effective theory of superconductivity can further be simplified and reformulated in terms of lattice gauge models [6, 8, 9, 10]. Thus, the $t$ - $J$ model (10) is related to a compact Abelian gauge model with internal symmetry (2), which couples holons and spinons. As in usual BCS superconductivity, under appropriate conditions the spinons couple and form bosonic quasiparticles. In a mean field theory one can define fields which behave under the gauge transformations (2) as:

$$
\begin{aligned}
\chi_{i j} & =\sum_{\sigma}\left\langle f_{i \sigma}^{\dagger} f_{j \sigma}\right\rangle \rightarrow \chi_{i j} \cdot \mathrm{e}^{-i\left(\alpha_{i}-\alpha_{j}\right)}, \\
\Delta_{i j} & =\left\langle f_{i \uparrow} f_{j \downarrow}-f_{i \downarrow} f_{j \uparrow}\right\rangle \rightarrow \Delta_{i j} \cdot \mathrm{e}^{i\left(\alpha_{i}+\alpha_{j}\right)} .
\end{aligned}
$$

The phase of the field $\chi$ is the compact $U(1)$ gauge field, $\theta_{i j} \equiv \arg \chi_{i j} \rightarrow \theta_{i j}+(\mathrm{d} \alpha)_{i j}$ with $(\mathrm{d} \alpha)_{i j}=\alpha_{j}-\alpha_{i}$, and the radial part, $\chi=\left|\left\langle\chi_{i j}\right\rangle\right|$, is the so-called "resonating valence bond" (RVB) coupling. The doubly-charged spinon-pair field $\Delta$ is an analog of a Cooper pair.

At high temperature the RVB coupling is vanishing, $\chi=0$, and the system is in the Mott insulator (or "poor metallic") phase. With decreasing temperature $\chi$ becomes non-zero, eventually enabling the formation of a spinon-pair condensate $\Delta=\left|\left\langle\Delta_{i j}\right\rangle\right|$ and/or of a holon condensate $b=\left\langle b_{i}\right\rangle[\underline{5}]$. Therefore, four phases [8, [9, 10] may emerge: the Fermi liquid (FL) phase with $b \neq 0$, $\Delta=0$, the spin gap (SG) phase with $b=0, \Delta \neq 0$, the superconductor (SC) phase with $b \neq 0, \Delta \neq 0$, and the strange metallic (SM) phase with $b=0, \Delta=0$. 
A compact Abelian two-Higgs model (cA2HM) in three dimensions with a $U(1)$ gauge link field $\theta$, a singlycharged holon field $\Phi_{1}$, and a doubly-charged spinon-pair field $\Phi_{2}$ is a feasible phenomenological model to describe the phase structure of the two-dimensional spin-charge separated quantum system [8, 9]. This model suffers from several limitations 2] to describe high- $T_{c}$ materials in the underdoped regime, since the model includes only Gaussian fluctuations about the mean field theory of the $t-J$ model and does not reflect the $S U(2)$ particle-hole symmetry at half-filling. However, it seems plausible that the cA2HM can be applied in the less-studied overdoped regime (called sometimes the SM region [2, 11]) where the particle-hole symmetry is explicitly broken.

Since high- $T_{c}$ materials are type- 2 superconductors, we restrict ourselves to the London limit in which the radial parts of both Higgs fields $\Phi_{Q}=\left|\Phi_{Q}\right| e^{i \varphi_{Q}}$ are frozen, $\left|\Phi_{1,2}\right|=$ const. The action of the model is defined as

$S_{\mathrm{A} 2 \mathrm{HM}}=-\beta \sum_{P} \cos \theta_{P}-\sum_{Q=1}^{2} \kappa_{Q} \sum_{l} \cos \left(\mathrm{d} \varphi_{Q}+Q \theta\right)_{l},(5)$

where $\theta_{P}$ is the standard lattice plaquette. The model (5) obeys a lattice version of the $U(1)$ internal gauge symmetry (2): $\theta \rightarrow \theta+\mathrm{d} \alpha, \varphi_{Q} \rightarrow \varphi_{Q}-Q \alpha$. It describes the hole (electron) "constituents" by the dynamical holon $\varphi_{1}$ and spinon $\varphi_{2}$ phases, which strongly interact via the dynamical gauge field $\theta$. The inverse gauge coupling $\beta$ in Eq. (5), $\beta=\chi_{f}+\chi_{b}$, is given by the diamagnetic susceptibilities $\chi_{f}$ of the spinon and $\chi_{b}$ of the holon fields. The hopping parameters $\kappa_{Q}$ are connected to the doping concentration $x$ and the couplings $t$ and $J$ of (11) as follows: $\kappa_{1} \propto t \cdot x$ and $\kappa_{2} \propto J$ [2].

Multi-Higgs models similar to Eq. (5) appear not only for theories of high- $T_{c}$ superconductivity but also in models for metallic hydrogen [12] (using non-compact gauge fields and $Q_{1}=-Q_{2}=2$ ) and for multi-band superconductors 13.

The model (5) contains three kinds of topological defects: a monopole and two types of vortices, referred to as the holon and the spinon vortex [2, [8]. The monopole has magnetic charge $2 \pi \hbar$ while the holon (spinon) vortex carries magnetic flux quanta $2 \pi \hbar(\pi \hbar)$ of the gauge field $\theta$. Since the magnetic flux is conserved, one monopole is simultaneously a source of one holon vortex and two spinon vortices. The defects can be best studied in the dilute state characterizing the superconducting phase of the model with nonvanishing condensates. In this phase, the core of the holon (spinon) vortex is in the FL (SG) phase. The monopole core is in the SM phase where both condensates are zero.

The structure of the three-dimensional phase diagram of the model (5) is quite complicated. However, the faces and edges of the $3 D$ phase "cube" (i.e. the limiting cases of vanishing or large couplings $\beta$ and $\kappa_{1,2}$ ), respectively, can be related to various well-known and sometimes non- trivial systems. These limiting cases are shown schematically on the faces of the $3 D$ phase cube presented in Fig. 1(a) and are discussed in detail below. The interior of the cube in Fig. 1(a) is our qualitative prediction of the phase structure of the cA2HM. The shaded $\left(\kappa_{1}, \kappa_{2}\right)$ plane should be compared with Fig. T(b) which represents the result of our numerical investigation for $\beta=1.0$. The numerical result is consistent with the prediction and will be presented later.

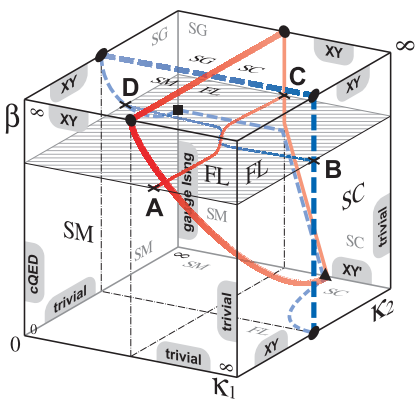

(a)

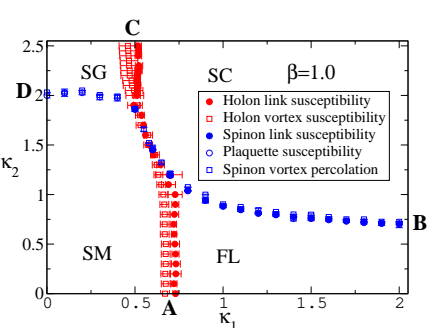

(b)
FIG. 1: (a) The qualitative $3 D$ phase diagram and (b) its numerically obtained $2 D$ cross-section at $\beta=1$.

On the $\kappa_{2}=0$ face the compact Abelian $Q=1$ Higgs model $\left(\mathrm{cAHM}_{Q=1}\right)$ emerges. There the holon condensate $b$ and the holon vortices are controlled by the compact $U(1)$ gauge field. The phase of the spinon-pair field is disordered which implies condensation of the spinon vortices and, therefore, vanishing $\Delta$. In the $\left(\beta, \kappa_{1}\right)$ plane two phases [14, 15, 16, 17] exist: (i) the broken/Higgs phase with $b \neq 0$ at large $\beta /$ large $\kappa_{1}$ corresponding to the FL phase, and (ii) the confining/symmetric phase with $b \approx 0$ at small $\beta /$ small $\kappa_{1}$. The confining phase is the SM phase with some properties of the FL phase. The holon condensate is nowhere exactly vanishing because the phases are connected analytically. The holon vortices are almost massless and therefore dense in the SM phase, and they become heavy and dilute in the FL phase. At the $\kappa_{1}=0$ edge the model reduces to the compact $U(1)$ theory which is confining at any $\beta$ due to the monopole plasma [18]. The plasma destroys the holon condensate, $b=0$. At the edge $\beta \rightarrow \infty$ the model becomes the $3 D X Y$ model with a second order transition [19] at $\kappa_{1, c}=\kappa_{c}^{X Y} \approx 0.45420 \ldots$ Since away from this limit a relevant global symmetry is absent, no singularities in the thermodynamic quantities are expected. With decreasing $\beta$, the SM and FL phases, being analytically connected, can be thought to be separated by a Kertész line [20], across which the holon condensate is a smooth quantity.

On the face $\kappa_{2} \rightarrow \infty$ the model (5) reduces to a $3 D$ $X Y-\mathbb{Z}_{2}$ model with the action

$$
S_{X Y-\mathbb{Z}_{2}}=-\beta \sum_{P} \sigma_{P}-\kappa_{1} \sum_{l} \sigma_{l} \cos (d \varphi)_{l} .
$$


This model describes a $X Y$-type matter field $\varphi=\varphi_{1}-$ $\varphi_{2} / 2$ which interacts via exchange of a $\mathbb{Z}_{2}$ gauge field $\sigma_{l}= \pm 1$. The model - studied numerically in Ref. 21] - contains three phases which, according to the adopted classification, are: (i) the SM phase at small $\beta /$ small $\kappa_{1}$, (ii) the SG phase at large $\beta /$ small $\kappa_{1}$ and (iii) the $\mathrm{SC}$ phase at large $\beta /$ large $\kappa_{1}$.

For not too large $\kappa_{1}$ the SM and SG phases are separated by a second order phase transition of the Ising universality class across a line $\beta_{c}\left(\kappa_{1}\right)$ with the $3 D$ gauge Ising (gI) model limit [22]: $\beta_{c}(0)=\beta_{c}^{\mathrm{gI}} \approx 0.7613 \ldots$ At large $\beta$ the SG phase and SC phases are separated by a second order phase transition in the $3 D X Y$ universality class, $\lim _{\beta \rightarrow \infty} \kappa_{1, c}(\beta)=\kappa_{c}^{X Y}$. Inside the phase diagram these transition lines merge and continue as a single second order transition in the $X Y$ universality class along a line $\kappa_{1, c}(\beta)$ which ends at the $\beta=0$ edge in $\kappa_{1, c}(0)=\kappa_{c}^{X Y^{\prime}} \approx 1.6$. Here the model is reduced to a (modified) $X Y$ model with the action $S_{X Y^{\prime}}=$ $-\sum_{l} \log \cosh \left[\kappa_{1} \cos (\mathrm{d} \varphi)_{l}\right]$.

The model (6) has exotic phases predicted for strongly correlated electron systems [23] and it is characterized by so-called "visons" - fractionally charged excitations related to the presence of $\mathbb{Z}_{2}$ degrees of freedom. A vison coincides with the spinon vortex at $\kappa_{2} \rightarrow \infty$, while the holon vortex turns into a $X Y$ vortex. The $X Y$ phase angle $\varphi$ becomes the phase of the so-called "chargon" particle. The SG phase corresponds to the fractionalized phase where visons are absent and chargons are free particles. In the SM phase the visons are condensed, and chargons are confined. The SC phase corresponds to a superfluid state where both visons and $X Y$ vortices are dilute and chargons are free.

On the $\kappa_{1}=0$ face the holon condensate vanishes, $b=0$, and the spinon-pair condensate $\Delta$ is described by the $\mathrm{cAHM}_{Q=2}$ model. The phase diagram in the $\left(\beta, \kappa_{2}\right)$ plane can be deduced from results of Ref. [14, 24]: The SM phase is located at small $\beta /$ small $\kappa_{2}$, while the SG phase is residing in the large $\beta /$ large $\kappa_{2}$ corner. The phases are separated by a second order phase transition line which ends at $\beta_{c}=\beta_{c}^{\mathrm{gI}}$ in the large $\kappa_{2}$ limit and at $\kappa_{2, c}=\kappa_{c}^{X Y}$ in the large $\beta$ limit.

On the $\kappa_{1} \rightarrow \infty$ face the model (5) reduces to a $X Y$ model, $S_{X Y}=-\kappa_{2} \sum_{l} \cos (\mathrm{d} \varphi)_{l}, \varphi=2 \varphi_{1}+\varphi_{2}$, which controls the spinon condensate. Due to the constraint $\theta=\mathrm{d} \varphi_{1}+2 \pi l, l \in \mathbb{Z}$, the holon vortices are suppressed and therefore $b \neq 0$ in the whole $\left(\beta, \kappa_{2}\right)$ plane. The phase diagram is divided by a straight line of a second order $X Y$-like transition parallel to the $\beta$ axis at $\kappa_{2, c}(\beta)=$ $\kappa_{c}^{X Y}$. This line separates the FL phase (with condensed spinon vortices and $\Delta=0)$ at $\kappa_{2}<\kappa_{c}^{X Y}$ from the SC phase (with suppressed spinon vortices and $\Delta \neq 0$ ).

On the $\beta=0$ face we obtain a two-Higgs system interacting ultra-locally via a non-propagating gauge field. After integrating out the gauge field in the Villain representation [25], one gets a $X Y$ model with the effective
$X Y$-coupling, $\kappa_{\text {eff }}^{V}=\kappa_{1}^{V} \kappa_{2}^{V} /\left(\kappa_{1}^{V}+4 \kappa_{1}^{V}\right)$ where the superscript $V$ indicates the Villain coupling. The phase diagram in the $\left(\kappa_{1}, \kappa_{2}\right)$ plane contains two phases: the $\mathrm{SC}$ phase with non-zero condensates $\Delta$ and $b$ in the large $\kappa_{1} /$ large $\kappa_{2}$ corner and a SM-FL phase in the remaining part of the phase diagram. The phases are separated by a second order $X Y$-type phase transition which starts at $\kappa_{1, c}=\kappa_{c}^{X Y^{\prime}}$ at the $\kappa_{2} \rightarrow \infty$ edge and ends at $\kappa_{2, c}=\kappa_{c}^{X Y}$ for $\kappa_{1} \rightarrow \infty$. At these two edges the model is reduced to a modified and a usual $X Y$ model, respectively. The SMFL phase is actually the FL phase at large $\kappa_{1}$, where the holon condensate is non-vanishing, and the $S M$ phase at large $\kappa_{2}$, where the holon condensate tends to zero. Those phases are analytically connected, similar to the $\kappa_{2}=0$ face considered above.

On the $\beta \rightarrow \infty$ face the system reduces to two decoupled $X Y$ models describing the holon and spinon superfluidities. The phase diagram in $\left(\kappa_{1}, \kappa_{2}\right)$ includes all SM, SG, FL, SC phases as shown on the upper face of Fig. 1(a). The predicted $3 D$ phase diagram implies, in particular, that at sufficiently strong gauge coupling, $\beta<\beta_{c}^{\mathrm{gI}}$, the SG phase ceases to exist.

Performing Monte Carlo simulations we have investigated the phase diagram of the cA2HM. We made an exploratory first study on a $16^{3}$ lattice, choosing three values of the gauge couplings, $\beta=1.0,1.5$ and 2.0 , on a dense grid of points spanning the $\left(\kappa_{1}, \kappa_{2}\right)$ hopping parameter plane over the range $0<\kappa_{1,2} \leqslant 2.0,2.5$. In all figures we show the results for the strong coupling $\beta=1.0$ only.

The density of the monopoles, $\rho_{\text {mon }}$, is plotted in the upper panel of Fig. 2 over the $\left(\kappa_{1}, \kappa_{2}\right)$ plane. With increasing hopping parameters $\kappa_{1}$ or $\kappa_{2}$ the monopole density gets suppressed. As shown in the middle panel of Fig. 2 the density of the holon vortices $\rho_{\text {vort }}^{(1)}$ (spinon vortices $\left.\rho_{\text {vort }}^{(2)}\right)$ significantly drops down with increasing $\kappa_{1}$ $\left(\kappa_{2}\right)$.

The connectivity $C_{\mathrm{IR}}^{(1,2)}=C^{(1,2)}\left(R_{\max }\right)$ of the vortex clusters is derived from the cluster correlation functions

$$
C^{(1,2)}(|\vec{R}|)=\frac{\left\langle\sum_{\vec{x}, \vec{y}} \sum_{c} \Theta_{c}(\vec{x}) \Theta_{c}(\vec{y}) \delta(\vec{R}-\vec{x}+\vec{y})\right\rangle}{\sum_{\vec{x}, \vec{y}} \delta(\vec{R}-\vec{x}+\vec{y})}
$$

$\left(\Theta_{c}(\vec{x})=1\right.$ or 0 is the characteristic function of the vortex cluster $c, R_{\max }$ the maximal distance on the periodic lattice) gives a clear view of the phase diagram of the model [17, 20, 26]. Any Higgs condensate suppresses the proliferation of the corresponding vortices: they are prevented to percolate over infinitely long distances. We show the connectivities $C_{\mathrm{IR}}^{(1,2)}$ for the spinon (holon) vortex clusters in the lower panel of Fig. 22 The corresponding phase diagram is shown in Fig. 1(b). It agrees with the cross-section through the $3 D$ phase cube marked by the shaded plane in Fig. 1(a). We plotted letters $A, B, C$ and $D$ in Figs. 1(a) and (b) to identify the same points.

To clarify the nature of the phase transitions, we studied the volume dependence of the average plaquette and 


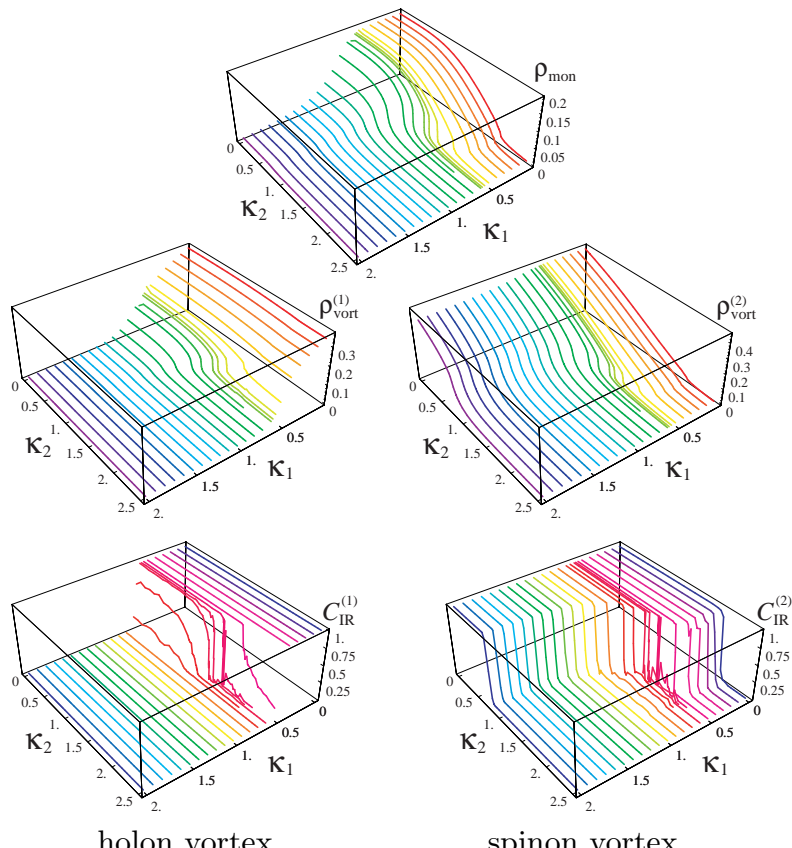

FIG. 2: The monopole density (top), the vortex densities (middle), and the vortex connectivities (bottom). Left: holon vortices; right: spinon vortices.

both link contributions to the action (5) as well as their susceptibilities, respectively, in different regions of the phase diagram at $\beta=1.0$. Fig. B (left) clearly exempli-
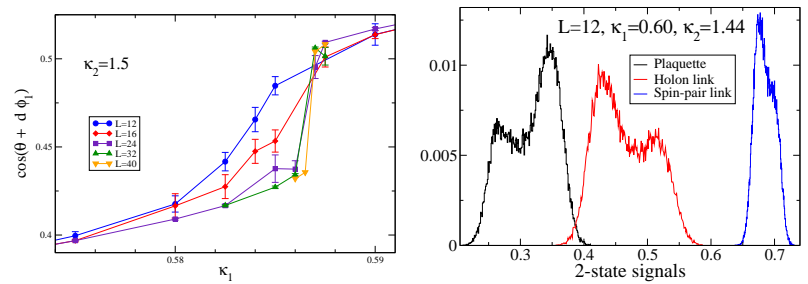

Holon link expectation value Histograms of action parts

FIG. 3: Signatures for the first order transition.

fies the first order nature of the transition (observed in all parts of action) by the jump developing in the holon link vs. $\kappa_{1}$ at fixed $\kappa_{2}$ in the crossing region of the transition lines. Already at lattice size $32^{3}$ we do not observe tunnelings for the selected $\kappa_{1}$ values within $5 \times 10^{5}$ iterations. In Fig. 3 (right) we present typical two-state signals (for a $12^{3}$ lattice) of all three terms in the action at $\left(\kappa_{1}, \kappa_{2}\right)$ near the transition. The two-state signal with respect to the plaquette and the holon link becomes very weak when one goes to smaller $\kappa_{1}$ along the (except for the direct transition between the SM and the SC phases) horizontal dark-dotted (blue) line.

It is known that for $\kappa_{1} \rightarrow 0$ the transition vs. $\kappa_{2}$ is of second order. Similarly, for large $\kappa_{1}$ the transition is most likely of second order, again in the $X Y$ universality class. We found that for the largest volumes $40^{3}-48^{3}$ under study the increase of the spinon link susceptibility at $\kappa_{1}=2.0$ stops as function of the lattice size as expected for the $X Y$ model at $\kappa_{1} \rightarrow \infty$ 27]. Selecting two $\kappa_{2}$ values outside the crossing regime, where one of the transition lines (the light-dotted [red] one) runs vertical, we observe that there is no thermodynamic transition vs. $\kappa_{1}$ for the smaller $\kappa_{2}$, in agreement with what could be anticipated from the limit $\kappa_{2} \rightarrow 0$.

Summarizing, we predicted the qualitative $3 D$ phase diagram of the cA2HM proposed as an effective model for high- $T_{c}$ cuprates in the overdoped regime. At strong gauge coupling (however $\beta>\beta_{c}^{\mathrm{gI}}$ ) we found two transitions associated with the pattern of vortex percolation and identified the Fermi liquid, spin gap, superconductor and strange metallic phases in agreement with the proposed phase diagram. The percolation transitions are accompanied with thermodynamic phase transitions except for small holon hopping parameter. First hints for a changing order along the thermodynamic transition lines are found. In the region where the two transition lines merge, a direct phase transition between the SC and SM phases exists and is found to be first order.

E.-M.I. is supported by the DFG Forschergruppe 465 "Gitter-Hadronen-Phänomenologie". M.N.Ch. is supported by a STINT Institutional grant IG2004-2 025 and by the grants RFBR 04-02-16079, 05-02-16306, DFG 436 RUS 113/739/0, and MK-4019.2004.2. M.N.Ch. is thankful to members of Department of Theoretical Physics of Uppsala University for kind hospitality and stimulating environment.

[1] E. Dagotto, Rev. Mod. Phys. 66 (1994) 763; P. W. Anderson et al., J. Phys. Cond. Matter 16, R755 (2004).

[2] P. A. Lee, N. Nagaosa, X.-G. Wen, cond-mat/0410445.

[3] P. W. Anderson, Science 235 (1987) 1196; F. C. Zhang, and T. Rice, Phys. Rev. B 37, 3759 (1988).

[4] S. E. Barnes, J. Phys. F 6 (1976) 1375; P. Coleman, Phys. Rev. B 29 (1984) 3035; X. G. Wen, F. Wilczek, A. Zee, Phys. Rev. B 39, 11413 (1989); C. Mudry, E. Fradkin, ibid, 49, 5200 (1994).

[5] G. Baskaran, Z. Zou, and P. W. Anderson, Solid State Comm. 63, 973 (1987).

[6] G. Baskaran, P. W. Anderson, Phys. Rev. B 37 (1988) R580.

[7] L. D. Faddeev, A. J. Niemi, Phys. Lett. B 464, 90 (1999); A. J. Niemi, N. R. Walet, Phys.Rev. D72, 054007 (2005); A. J. Niemi, hep-ph/0510288 M. N. Chernodub, hep-th/0506107

[8] P. A. Lee, N. Nagaosa, Phys. Rev. B 45 (1992) 000966; ibid. 46 (1992) 005621.

[9] N. Nagaosa, P. A. Lee, Phys. Rev. B 61 (2000) 9166.

[10] I. Ichinose, T. Matsui, Phys. Rev. B 51, 11860 (1995); I. Ichinose, T. Matsui, M. Onoda, ibid. 64 104516, (2001).

[11] C. M. Varma, Phys. Rev. B 55, 14554 (1997); C. Castellani, C. D. Castro, M. Grilli, Z. Phys. 103, 137 (1997); J. L. Tallon, J. W. Loram, Physica C 349, 53 (2000). 
[12] E. Babaev, A. Sudbo, N.W. Ashcroft, Nature 431, 666 (2004); E.Babaev, L.D.Faddeev, A.J.Niemi, Phys. Rev. B 65, 100512 (2002); E.Babaev, ibid. 89, 067001 (2002).

[13] J. Smiseth, E. Smorgrav, A. Sudbo, Phys. Rev. Lett. 93, 077002 (2004); E. Smorgrav et al. ibid. 94, 096401 (2005); J. Smiseth et al., Phys. Rev. B 71, 214509 (2005).

[14] E.H.Fradkin, S.H.Shenker, Phys. Rev. D 19 (1979) 3682.

[15] K. Osterwalder, E. Seiler, Annals Phys. 110 (1978) 440.

[16] M. B. Einhorn and R. Savit, Phys. Rev. D 17, 2583 (1978); ibid. 19, 1198 (1979).

[17] M. N. Chernodub, E.-M. Ilgenfritz, A. Schiller, Phys. Lett. B 547, 269 (2002).

[18] A. M. Polyakov, Nucl. Phys. B 120 (1977) 429.

[19] A. P. Gottlob, M. Hasenbusch, Physica A 201, 593 (1993); G. Kohring, R. E. Shrock, P. Wills, Phys. Rev. Lett. 57 (1986) 1358.
[20] S. Wenzel et al., Phys. Rev. Lett. 95 (2005) 051601; hep-lat/0510099

[21] R. D. Sedgewick, D. J. Scalapino and R. L. Sugar, Phys. Rev. B 65, 54508 (2002).

[22] R. Balian, J. M. Drouffe and C. Itzykson, Phys. Rev. D 11, 2098 (1975).

[23] T. Senthil, M. P. A. Fisher, Phys. Rev. B 62, 7850 (2000); O. I. Motrunich, T. Senthil; Phys. Rev. Lett. 89, 277004 (2002).

[24] J. Smiseth et al., Phys. Rev. B 67 (2003) 205104.

[25] J. Villain, J. Phys. (France) 36, 581 (1975).

[26] M. N. Chernodub et al., Phys. Rev. D 71, 074502 (2005); Phys. Lett. B 605, 161 (2005).

[27] M. Campostrini et al., Phys. Rev. B 63, 214503 (2001). 